Check for updates

Cite this: RSC Adv., 2019, 9, 30641

Received 23rd July 2019

Accepted 13th September 2019

DOI: $10.1039 / c 9 r a 05675 k$

rsc.li/rsc-advances

\section{A tri-phase percolative ceramic composite with high initial permeability and composition- independent giant permittivity}

\author{
Wei Tian, $\uparrow^{\mathrm{a}}$ Bin Xiao, $\uparrow^{\mathrm{ab}}$ Zuhuang Chen, ${ }^{\mathrm{a}}$ Yu Tang, (D) ac Ning Ma, ${ }^{\mathrm{a}}$ Zongrong Wang ${ }^{\star a}$ \\ and Piyi Du (D) *a
}

The drastic change of properties near the percolation threshold usually limits the practical applications of percolative composite materials. In this work, a tri-phase system, i.e. a $\mathrm{BaTiO}$ (BTO) $/ \mathrm{Ni}_{0.5} \mathrm{Zn}_{0.5} \mathrm{Fe}_{2} \mathrm{O}_{4}$ (NZFO)/BaFe ${ }_{12} \mathrm{O}_{19}$ (BFO) ceramic composite, is proposed and investigated in detail. The BFO phase was in situ formed during a hybrid process of sol-gel and self-combustion methods. The content of the BFO phase could be tuned conveniently by controlling the preparation conditions. The as-prepared BTO/ NZFO/BFO tri-phase composite exhibited unprecedented stable dielectric properties that were distinct from those of conventional percolative composites above the percolation threshold due to the existence of a third phase. When the volume fraction of the NZFO phase exceeds $55 \%$, the electrical conductivity and effective permittivity of the composite remain at a stable value of about $10^{-5} \mathrm{~S} \mathrm{~cm}^{-1}$ and 10000 , respectively, which is almost independent of the composition. Such behavior is the result of the synergistic control effect of the percolation effect and specific phase composition in the system. It is evident that the stability of the dielectric properties of the composite is chiefly contributed by the introduction of the BFO phase. Meanwhile, the composite exhibited a relatively high permeability of $\sim 17$ with $90 \%$ NZFO loading, and its saturated magnetization is larger than $73 \mathrm{emu} \mathrm{g}^{-1}$, approximately $95 \%$ of the pure NZFO phase. The finding of our BTO/NZFO/BFO tri-phase ceramic composite with stable giant permittivity and extremely high permeability paves a new way to solve the difficulty of property instability above the percolation threshold in the utilization of percolative materials.

\section{Introduction}

Many efforts have been devoted to the investigation of multiferroic materials both experimentally and theoretically due to their capability to satisfy the fast growing demands in advanced electronic devices, such as memory devices with multi-state data storage, solid-state transformers and high sensitivity magnetic field sensors. ${ }^{1-6} \quad$ Ferroelectric/ferromagnetic composite materials are potential candidates to meet the requirements of miniaturization and multi-functionality in next-generation electronic devices. For example, integrated capacitance-inductance elements and anti-electro-magnetic interference (anti-EMI) filters usually require more space saving in circuit boards or replacement of passive filters

${ }^{a}$ State Key Laboratory of Silicon Materials, School of Materials Science and Engineering, Zhejiang University, Hangzhou, 310027, China. E-mail: dupy@zju.edu. cn; zrw@zju.edu.cn

${ }^{b}$ Department of Materials Science and Engineering, Shenzhen Engineering Research Center for Novel Electronic Information Materials and Devices, Southern University of Science and Technology, Shenzhen, 518055, China

${ }^{c}$ Department of Materials Science and Engineering, College of Aerospace Science and Engineering, National University of Defense Technology, Changsha, 410073, China

$\dagger$ These authors contributed equally to this work. constructed from separate capacitance and inductance elements in order to realize the purpose of miniaturization and multi-functionality simultaneously. However, according to the composite law, the capacitance and inductance characteristics with respect to the corresponding constituent phases are dependent on their relative volume fraction in the system. In this scenario, the contribution of the ferroelectric phase would get inevitably undermined if the content of the ferromagnetic phase further increases, or vice versa. Once a nonmagnetic phase has been introduced into the system, the magnetic particles could be blocked or isolated, leading to lower permeability. The same situation may also occur when excessive ferromagnetic phase has been introduced into composite, giving rise to a decreased permittivity. Zhang's group reported a $\mathrm{BaTiO}_{3}-\mathrm{Ni}_{0.2} \mathrm{Cu}_{0.2} \mathrm{Zn}_{0.6} \mathrm{Fe}_{2} \mathrm{O}_{4} \quad$ ferroelectric/ferromagnetic composite prepared by solid phase synthesis method, neither the permittivity nor the permeability of which was satisfactorily high as anticipated although a combination of both dielectric and magnetic properties could be obtained. In particular, when $30 \% \mathrm{BaTiO}_{3}$ had been added, the permittivity of the composite was reduced to lower than 40 and the permeability was only around $10 .^{7}$ Therefore, limited by the composite law of such systems, it is still quite challenging to prepare composites with 
both satisfactorily high dielectric and magnetic properties. To solve this problem, materials scientists have tried to utilize percolation effect to avoid the negative influence of the composite law and have successfully obtained a number of percolative composites that could exhibit giant permittivity and high permeability simultaneously., ${ }^{\mathbf{8} 9}$ Representatively, Carlos Pecharromán et al. carried out many systematic studies involving the $\mathrm{Ni} / \mathrm{BaTiO}_{3}$ percolative system ${ }^{\mathbf{1 0}}$ and C. J. Won discovered that the $\mathrm{Ni}_{2} \mathrm{MnGa} / \mathrm{BaTiO}_{3}$ composite system also exhibited percolative behavior with a percolation threshold of 0.4. ${ }^{11}$ By varying the concentration and types of the ferrite fillers, it is feasible to prepare different percolative materials with diverse properties.

The debut of percolative composites undoubtedly casts light on the design of novel functional materials. However, some new problems are still unsolved for such systems. One of them involves the instability of properties near the percolation threshold $\left(f_{c}\right)$ as they usually exhibit a nonlinear behavior at this critical point. It is well known that the conductivity $(\sigma)$ and permittivity $(\varepsilon)$ of the composites would increase to a considerable extent following a scaling law near $f_{c}$, which is usually expressed as: $\mathbf{9 , 1 2}$

$$
\begin{gathered}
\sigma \propto \sigma_{\mathrm{D}}\left(f_{\mathrm{c}}-f\right)^{-q}, f<f_{\mathrm{c}} \\
\varepsilon=\varepsilon_{0}\left|\frac{f_{\mathrm{c}}-f}{f_{\mathrm{c}}}\right|^{-q}, f<f_{\mathrm{c}}
\end{gathered}
$$

where $f$ is the volume fraction of the ferromagnetic phase, $q$ the critical index, $\sigma_{\mathrm{D}}$ and $\varepsilon_{0}$ are the conductivity and permittivity of the ferroelectric matrix, respectively. In typical percolative composites, the momentum of dielectric properties would be waning obviously above the percolation threshold. Although the percolation threshold could be elevated to a larger value for some systems, ${ }^{13}$ it is still challenging to find the exact value of $f_{\mathrm{c}}$ and how to elevate it due to the complexity of physics in these composites, which usually leads to different values of $f_{\mathrm{c}}$ in different systems. ${ }^{\mathbf{1 3 , 1 4}}$ Obviously, the instability of properties in the vicinity of $f_{\mathrm{c}}$ is not acceptable for practical applications.

Generally, the permittivity and conductivity of a percolative composite will increase sharply and nonlinearly near percolation threshold $f_{\mathrm{c}}$, and their dependence on the volume fraction of the conductive phase near $f_{\mathrm{c}}$ abides by the scaling law as described by eqn (1) and (2). In conventional percolative NZFO/ BTO system, the NZFO ferrite phase is a typical semiconductor with lower conductivity compared with general percolative fillers such as metal particles. ${ }^{\mathbf{1 4}}$ It is well known that the percolation threshold is achieved once the conductive particles begin to connect with each other, leading to a steep increase in the permittivity and conductivity of the composites due to the formation of percolation paths. Therefore, if using semiconductive NZFO ferrite as the conductive phase, the percolation threshold of the composite will increase most probably to a higher value compared with those of conventional percolative composites. $^{13,15}$ Moreover, in the composite prepared by liquid enwrapping method, Ba-rich layers with relatively high resistivity would easily enwrap and separate the semiconductive NZFO particles, preventing the formation of conductive paths between the ferrite particles. As a result, the percolation threshold would generally shift to even higher values such as $f_{\mathrm{c}}$ $=0.8-0.9$ in this case..$^{\mathbf{1 3 , 1 6}}$

Our previous work revealed that the BTO/NZFO composite is a typical percolative system with super high percolation threshold and resultant giant permittivity and high permeability. ${ }^{\mathbf{1 3 , 1 5 , 1 7 , 1 8}}$ It should be pointed out that due to the fast nonlinearly increment in the electrical properties in the vicinity of the percolation threshold, some inconvenience will be brought to the practical applications of the composite materials. The inability of properties is a big problem especially under the circumstances where huge change in properties is not acceptable.

Hereby, we propose a feasible way of solving the difficulties of property instability near the percolation threshold of percolative composites. For the BTO/NZFO composite system, the ionic interdiffusion or chemical reactions are exploited during the sintering process to facilitate the formation of additional phase such as barium ferrite $\left(\mathrm{BaFe}_{12} \mathrm{O}_{19}\right)$ in the composite, a commonly used microwave absorbing materials, has also been widely investigated. ${ }^{19-23}$ With the formation of this phase, a Debye platform appears in the permittivity-frequency curve of the composite, which is quite distinctive from that of traditional percolative ceramic composites especially at low frequency. In this work, we report the impressively stable dielectric and magnetic properties of the $\mathrm{BaTiO}_{3} / \mathrm{Ni}_{0.5} \mathrm{Zn}_{0.5} \mathrm{Fe}_{2} \mathrm{O}_{4}$ ceramic composite coexisting with the $\mathrm{BaFe}_{12} \mathrm{O}_{19}$ phase. The physical mechanism behind this behavior is also investigated in-depth theoretically.

\section{Experimental}

\section{Samples preparation}

(i) The preparation of Nickel-Zinc ferrite powder. NickelZinc ferrite powder was prepared by self-combustion method with iron nitrate $\left(\mathrm{Fe}\left(\mathrm{NO}_{3}\right)_{3} \cdot 9 \mathrm{H}_{2} \mathrm{O}\right)$, zinc nitrate $\left(\mathrm{Zn}\left(\mathrm{NO}_{3}\right)_{2}\right.$ $\left.\cdot 6 \mathrm{H}_{2} \mathrm{O}\right)$, nickel nitrate $\left(\mathrm{Ni}\left(\mathrm{NO}_{3}\right)_{2} \cdot 6 \mathrm{H}_{2} \mathrm{O}\right)$ as starting materials and glycine and deionized water as solvents. The composition of $\mathrm{Ni}-\mathrm{Zn}$ ferrite was fixed as $\mathrm{Ni}_{0.5} \mathrm{Zn}_{0.5} \mathrm{Fe}_{2} \mathrm{O}_{4}$. The $80.8 \mathrm{~g}$ iron nitrate, $14.9 \mathrm{~g}$ zinc nitrate and $14.5 \mathrm{~g}$ nickel nitrate (according to stoichiometric ratio of $\mathrm{Ni}_{0.5} \mathrm{Zn}_{0.5} \mathrm{Fe}_{2} \mathrm{O}_{4}$ ) were dissolved into deionized water and $33.4 \mathrm{~g}$ glycine was added as combustion promoter. After being stirred for $2 \mathrm{~h}$, the precursor solution was obtained. The solution was heated in an oven until it spontaneously combusted and turned into puffed brown powder finally. The powder was ground and sieved for the next step.

(ii) The preparation of barium titanate solution. Acetic acid $\left(\mathrm{CH}_{3} \mathrm{COOH}\right)$, barium acetate $\left(\mathrm{Ba}\left(\mathrm{CH}_{3} \mathrm{COO}\right)_{2}\right)$, tetrabutyl titanate $\left(\mathrm{Ti}\left(\mathrm{OC}_{4} \mathrm{H}_{9}\right)_{4}\right)$ and ethylene glycol monomethyl ether $\left(\mathrm{CH}_{3}-\right.$ $\mathrm{OCH}_{2} \mathrm{CH}_{2} \mathrm{OH}$ ) were used as raw materials to prepare barium titanate solution. $25.5 \mathrm{~g}$ Barium acetate was initially dissolved with small amount of acetic acid under heating and stirring, and then cooled naturally to room temperature. $34.0 \mathrm{~g}$ tetrabutyl titanate was dissolved in ethylene glycol monomethyl ether to form precursor solution and then mixed with the aforementioned barium acetate solution in order to prepare barium titanate solution. 
(iii) The preparation of $x N Z F O /(1-x)$ BTO. The Ni-Zn ferrite powder prepared by self-combustion method was poured into as-prepared solution slowly according to the chemical formula of $\mathrm{xNZFO} /(1-x)$ BTO with a series of molar ratio $x=1.0,0.9$, $0.8,0.7,0.5,0.3,0.1$ and dispersed ultrasonically to avoid the agglomeration of the NZFO particles. During this process, the NZFO particles will be surrounded by barium titanate sol and turn into gel ultimately. The gel was dried and pre-sintered at $600{ }^{\circ} \mathrm{C}$ for $1 \mathrm{~h}$ to obtain the composite powder. The pre-sintered powder was added 5\% PVA, ground and sieved with 600 mesh for granulation. The powder was pressed into annular under an uniaxial pressure of $200 \mathrm{MPa}$ and sintered at $1300{ }^{\circ} \mathrm{C}$ for $10 \mathrm{~h}$, then naturally cooled down to room temperature.

\section{Characterization}

The density of the samples were measured and calculated using conventional Archimedes method. The surfaces of the samples were polished for XRD observation and phase analysis (RIGAKUD/MAX-C type X-ray diffractometer, $\mathrm{Cu} \mathrm{K} \alpha$, Tokyo, Japan.). SEM microscopy was performed to investigate in the microstructure and morphology of the composites using SIRION-type field emitting scanning electron microscope (Japan FEI Corporation). Before the test of electrical properties, the polished samples were cleaned ultrasonically and painted with silver electrodes. The dielectric properties were analyzed by Precision Impedance Analyzer (HP4294A-LRC) between $100 \mathrm{~Hz}$ and $1 \mathrm{MHz}$. The magnetic hysteresis loops were measured by Vibrating Sample Magnetometer (VSM, USA). The frequency dependence of permeability was measured by Agilent 16451B precision impedance analyzer (Palo Alto, CA) between $100 \mathrm{kHz}$ and $110 \mathrm{MHz}$.

\section{Results and discussion}

Fig. 1a shows the XRD patterns of the ceramic composite $x \mathrm{NZFO} /(1-x)$ BTO sintered at $1300{ }^{\circ} \mathrm{C}$ for $10 \mathrm{~h}$ with different molar contents of NZFO ferrite $(x=0.1,0.3,0.5,0.7,0.9)$. It can be seen that there are three constituent phases, i.e. NZFO, BTO and BFO, coexisting in the composite. The peak intensity of the BTO phase decreases gradually with the increase in $x$, which is opposite to the fast increment in that of the NZFO phase. Meanwhile, the peak intensity of the BFO phase also increases with increasing $x$ as shown in Fig. 1a. Fig. 1b shows the XRD patterns of the composite with $x=0.9$ sintered at $1300{ }^{\circ} \mathrm{C}$ for $1 \mathrm{~h}, 5 \mathrm{~h}$ and $10 \mathrm{~h}$, respectively. It demonstrates that the peak intensity of the BFO phase declines to a large extent with the prolongation of calcination time.

The XRD patterns show that the composite is constituted by perovskite BTO phase, magnetoplumbite BFO phase and spinel NZFO phase. Among these phases, the BFO phase is formed in situ and exists as a third constituent phase in the composite, leading to the formation of a completely new tri-phase composite. The formation of the BFO phase could be attributed to two mechanisms during the sintering process. The first one is the elemental interdiffusion and reactions between the ferroelectric BTO phase and the ferromagnetic NZFO phase, which would inevitably occur during the calcination process. ${ }^{\mathbf{1 4}}$ Since the formation of BFO needs more Fe source than Ba source, the increase in NZFO content would facilitate the generation of BFO in the composite. As a result, an increment in the volume content of BFO was observed with the increase of $x$, as illustrated in Fig. 1a. The second mechanism is the chemical reaction between $\mathrm{BaTiO}_{3}$ and $\alpha-\mathrm{Fe}_{2} \mathrm{O}_{3}$, which usually exists in BTO/NZFO composite that underwent a pre-sintering process. ${ }^{24}$ The formation of $\alpha-\mathrm{Fe}_{2} \mathrm{O}_{3}$ is closely related with NZFO content. In this case, as $x$ increases, abundant Fe source results in the formation of more $\alpha-\mathrm{Fe}_{2} \mathrm{O}_{3}$ and further more BFO due to more reactions between $\alpha-\mathrm{Fe}_{2} \mathrm{O}_{3}$ and the BTO phase in the final sintering process. It indicates that the existence of NZFO ferrite may promote the formation of BFO phase. Moreover, the content of BFO can be tuned easily by controlling the calcination time. As shown in Fig. 1b, longer sintering time would reduce the content of the $\mathrm{BFO}$ phase in the composite, which means the composition of the tri-phase composite is artificially controllable. The reduction in the peak intensity of BFO in the composite with longer duration supports the prediction that the BFO phase is formed via the elemental interdiffusion between BTO and NZFO, because the transportation of the constituent elements requires sufficient time, thus longer duration ensures the diffusion of the $\mathrm{Ba}$ and Fe elements into the interior of the
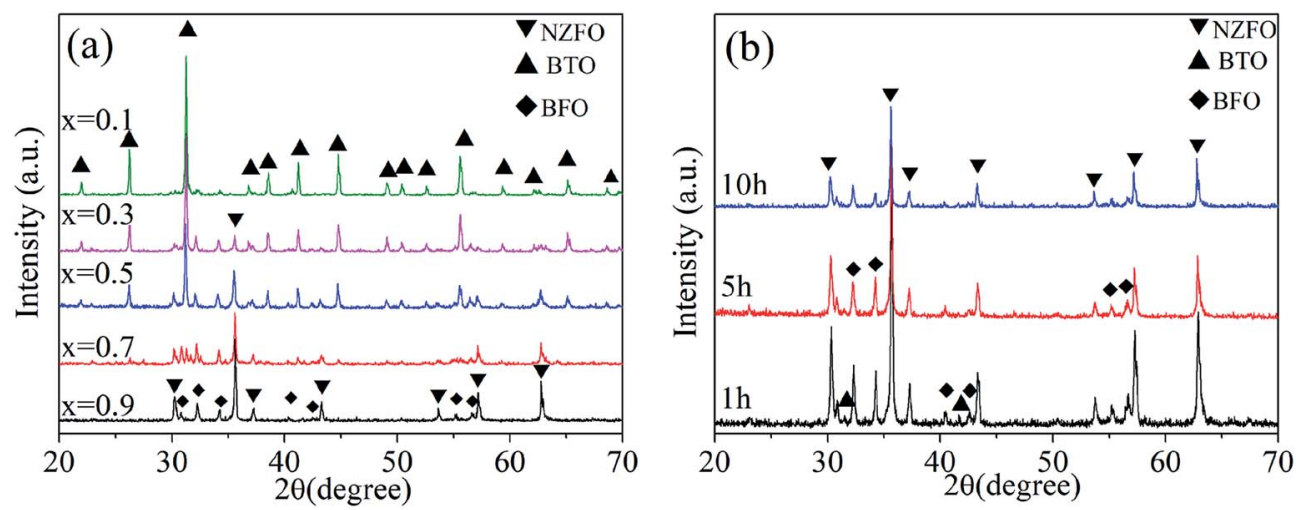

Fig. 1 (a) XRD patterns of the tri-phase ceramic composite with $x=0.1, x=0.3, x=0.5, x=0.7$ and $x=0.9$ sintered at $1300^{\circ} \mathrm{C}$ for $10 \mathrm{~h}$; (b) $\mathrm{XRD}$ patterns of the ceramic composite with $x=0.9$ sintered at $130{ }^{\circ} \mathrm{C}$ for $1 \mathrm{~h}, 5 \mathrm{~h}$ and $10 \mathrm{~h}$, respectively. 
crystals to form solid solution rather than form a third phase. More clearly, sufficiently long sintering time results in the diffusion of the constituent elements and finally the formation of the BTO and NZFO phase without BFO phase. It has been reported that if the duration is prolonged to $12 \mathrm{~h}$, only BTO and NZFO could be identified. ${ }^{14}$

Fig. 2 shows the density of the bi-phase BTO/NZFO ceramic composite sintered at $1200{ }^{\circ} \mathrm{C}, 1250{ }^{\circ} \mathrm{C}, 1280{ }^{\circ} \mathrm{C}$ and the triphase BTO/BFO/NZFO composite sintered at $1300{ }^{\circ} \mathrm{C}$ for $10 \mathrm{~h}$ with $x=0.7,0.8$ and 0.9 , respectively. It can be noticed that the composite has a low density when sintered at a relatively low temperature such as $1200{ }^{\circ} \mathrm{C}$. However, the density of the composite increases dramatically with increasing sintering temperature and reaches a maximum value at $1300{ }^{\circ} \mathrm{C}$ for $x=$ 0.7 and $x=0.8$. As for the composite with $x=0.9$, the highest density was obtained at $1280^{\circ} \mathrm{C}$ and a sharp decrease in density appears at $1300{ }^{\circ} \mathrm{C}$. Obviously, unlike the bi-phase composite, the density of tri-phase BTO/BFO/NZFO composite with $x=0.9$ exhibits a decrement at $1300{ }^{\circ} \mathrm{C}$ as shown in Fig. 2. This decrement is ascribed to the existence of the BFO phase, which has a lower density of only $5.28 \mathrm{~g} \mathrm{~cm}^{-3}$ compared with those of BTO $\left(5.86 \mathrm{~g} \mathrm{~cm}^{-3}\right)$ and NZFO $\left(5.33 \mathrm{~g} \mathrm{~cm}^{-3}\right)$ in the composite. ${ }^{25}$ Since the volume content of the BFO phase is more dominant at high ferrite loading as shown in Fig. 1a, the decrement in the density of the composite with $x=0.9$ can be well understood.

Fig. 3 shows the cross-sectional SEM images of the composite with $x=0.9$ sintered at $1200{ }^{\circ} \mathrm{C}, 1250{ }^{\circ} \mathrm{C}, 1280{ }^{\circ} \mathrm{C}$ and $1300^{\circ} \mathrm{C}$ for $10 \mathrm{~h}$, respectively. As can be seen, the microstructure seems porous and the grains are not well-crystallized at $1200{ }^{\circ} \mathrm{C}$. However, the grains grow larger with elevated sintering temperature and the microstructure becomes dense above $1280{ }^{\circ} \mathrm{C}$. The SEM image in Fig. 3d illustrates that the largest grain size and densest microstructure are simultaneously obtained at $1300{ }^{\circ} \mathrm{C}$, which seems inconsistent with the fact that its density exhibits a sharp decrease as shown in Fig. 2.

As mentioned above, concerning the theoretical density of BTO, BFO and NZFO, the density of the composite may decrease owing to the formation of the BFO phase in the composite. Experimentally, the BFO phase only forms at a high temperature such as $1300{ }^{\circ} \mathrm{C}$ with appropriate sintering duration $(10 \mathrm{~h}$ in this case). Meanwhile, the crystallinity and amount of the

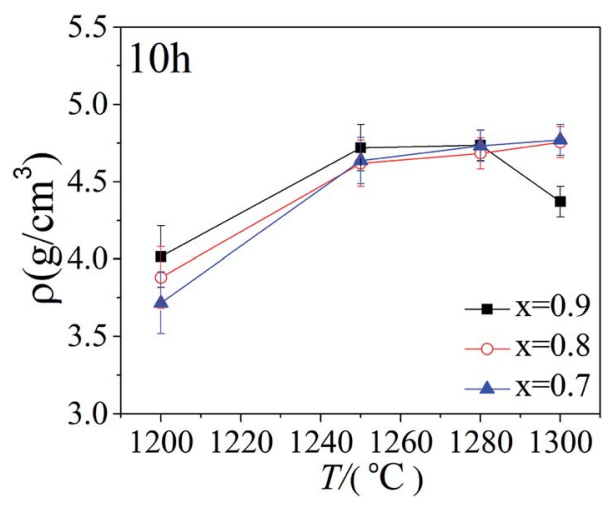

Fig. 2 Plots of the density of the ceramic composite sintered at $1200{ }^{\circ} \mathrm{C}, 1250{ }^{\circ} \mathrm{C}, 1280^{\circ} \mathrm{C}$ and $1300^{\circ} \mathrm{C}$ for $10 \mathrm{~h}$.
BFO phase in the composite decreases apparently with prolonging sintering time as shown in Fig. $1 \mathrm{~b}$.

It is reasonable to deduce that the BFO phase may originate from the chemical reaction that usually happens at a high temperature with proper duration. In general, sufficient sintering time assures the migration of the constituent elements in the composite to approach an equilibrium status. The BFO phase is most probably formed during the initial stage of the crystallization of the composite. The longer the reaction time is, the closer to the BTO/NZFO binary-equilibrium status will be. When the sintering time is prolonged to $12 \mathrm{~h}$ at $1300^{\circ} \mathrm{C}$, there is enough time for the chemical reaction to reach equilibrium state, where the BFO phase completely decomposed in the composite. ${ }^{\mathbf{1 3 , 1 4}}$ This is why only the BTO/NZFO binary composite was formed when sintered for $12 \mathrm{~h}$ in previous researches, ${ }^{\mathbf{1 4 , 2 6}}$ and it is also consistent with the gradual change of the peak intensity of the BFO phase as shown in Fig. 1b. In this work, a tri-phase composite comprised of BTO/NZFO/BFO could be formed at $1300{ }^{\circ} \mathrm{C}$ with a sintering duration of $10 \mathrm{~h}$.

Fig. 4a shows the frequency dependence of the effective permittivity of the tri-phase BTO/NZFO/BFO composite with different $x$ sintered at $1300{ }^{\circ} \mathrm{C}$ for $10 \mathrm{~h}$. The effective permittivity of the composite decreases with increasing frequency when $x$ is smaller than 0.5. However, it exhibits a wide plateau over several orders of frequencies in low frequency range above $x=0.5$. A rapid decrement and a low value of effective permittivity were observed in high frequency range for all samples, as shown in Fig. 4a. Fig. 4b shows the dielectric loss of the tri-phase composite. As can be seen, the peaks move to higher frequencies with the increase in NZFO content. The minimum dielectric loss of the tri-phase composite increases from 0.038 at $x=0.1$ to 0.607 at $x=0.9$.

The increment in dielectric loss can be attributed to enhanced Maxwell-Wagner effect caused by increased space charge polarization at interfaces such as phase boundaries and grain boundaries on the grounds that the introduction of BFO phase may lead to more interfaces in the composite. ${ }^{18,27}$ More interestingly, the effective permittivity of the tri-phase composite seems to keep constant above $x=0.5$ as shown in Fig. 4a. This behavior is quite different from that of binary BTO/ NZFO composite, whose effective permittivity usually increases nonlinearly with increased NZFO content. ${ }^{13}$ For clarity, the conductivity and effective permittivity of the tri-phase composite as a function of the volume fraction of NZFO ferrite $\left(f_{\mathrm{NZFO}}\right)$ at frequency of $100 \mathrm{~Hz}$ and $1 \mathrm{kHz}$ are provided in Fig. 5a and b, respectively. The molar content of NZFO ferrite $x$ $=0.1,0.3,0.5,0.7,0.8,0.9$ corresponds to the volume fraction of $f_{\mathrm{NZFO}}=0.118,0.342,0.548,0.738,0.829$ and 0.916 , respectively. It illustrates clearly that the conductivity and the effective permittivity of the composite increases rapidly until $f_{\mathrm{NZFO}}=$ 0.548 , and tends to maintain constant above it. The effective permittivity increases from only about $112\left(f_{\mathrm{NZFO}}=0.118\right)$ to $10340\left(f_{\mathrm{NZFO}}=0.548\right)$ at $100 \mathrm{~Hz}$ by almost two orders of magnitude. Correspondingly, the conductivity increases also by nearly two orders of magnitude from $1.74483 \times 10^{-7} \mathrm{~S} \mathrm{~cm}^{-1}$ at $f_{\mathrm{NZFO}}=0.118$ to $9.86525 \times 10^{-6} \mathrm{~S} \mathrm{~cm}^{-1}$ at $f_{\mathrm{NZFO}}=0.548$ at the same frequency. However, they preserve a stable value of 


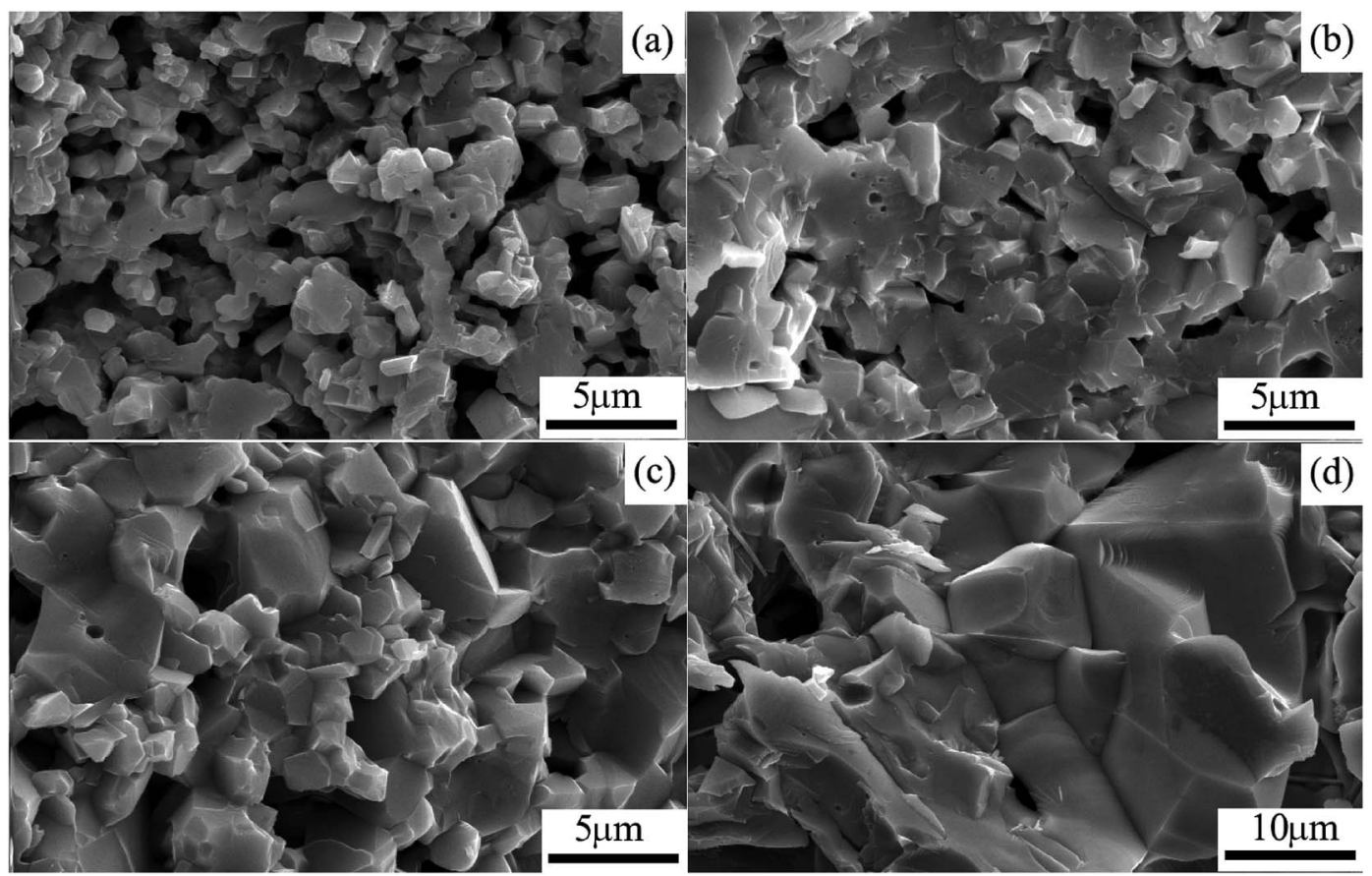

Fig. 3 Cross-sectional SEM images of the composite with $x=0.9$ sintered at (a) $1200{ }^{\circ} \mathrm{C}$, (b) $1250{ }^{\circ} \mathrm{C}$, (c) $1280{ }^{\circ} \mathrm{C}$ and (d) $1300{ }^{\circ} \mathrm{C}$ for $10 \mathrm{~h}$, respectively.

approximately 11000 for effective permittivity and $1 \times$ $10^{-5} \mathrm{~S} \mathrm{~cm}^{-1}$ for conductivity when the volume fraction of ferrite content is above $f_{\text {NZFO }}=0.548$.

It can be seen from Fig. 5 that the conductivity and effective permittivity of the tri-phase composite almost keeps constant above a certain point, i.e. $f_{\mathrm{NZFO}}=0.548$ in this case. This particular point is very close to the percolation threshold of the BTO/NZFO composite $\left(f_{\mathrm{c}}=0.55-0.65\right) .{ }^{26,28}$ In this work, the BFO phase is formed in situ due to the chemical reactions between BTO and NZFO, hence it most probably forms at the phase boundaries of the two phases. It is speculated that the percolation threshold of the $\mathrm{BTO} / \mathrm{NZFO} / \mathrm{BFO}$ composite is the same as that of the $\mathrm{BTO} / \mathrm{NZFO}$ composite, i.e. 0.55 in this case, and the following model could be used to interpret the atypical dielectric behaviour of the composite. Below $f_{\mathrm{c}}$, the matrix is insulating BTO phase, and the NZFO particles are dispersed uniformly in the matrix, thus the BFO phase is formed around the NZFO phase. However, above $f_{\mathrm{c}}$, the matrix is semiconducting NZFO phase with the BTO particles dispersed in it, hence the BFO phase is probably formed around the BTO phase.

In fact, if it possesses a core-shell structure, the BruggemanHanai approximation model (B-H model), which is expressed as below, can not be used to calculate the effective dielectric constant of the composite. ${ }^{13,15}$

$$
\left(1-f_{2}\right) \frac{K_{1}-K}{K_{1}+2 K}+f_{2} \frac{K_{2}-K}{K_{2}+2 K}=0
$$

where $K, K_{1}, K_{2}$ are the properties of the composite, the matrix and the filler, respectively. $f_{2}$ is the volume fraction of the filler phase. To simplify the calculation, we must consider the NZFO/ BFO and BTO/BFO as a whole below and above $f_{\mathrm{c}}$, respectively, by assuming that the equivalent filler 'particle' of NZFO/BFO and $\mathrm{BTO} / \mathrm{BFO}$ possess a core-shell structure where the BFO phase exists as the shell.
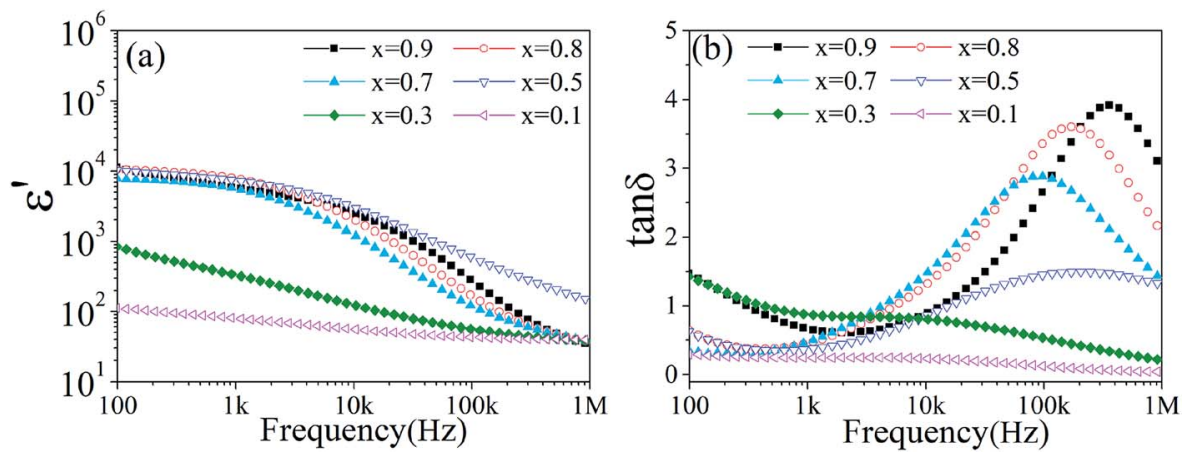

Fig. 4 (a) Effective permittivity and (b) dielectric loss of the tri-phase BTO/NZFO/BFO ceramic composite as a function of frequency. 

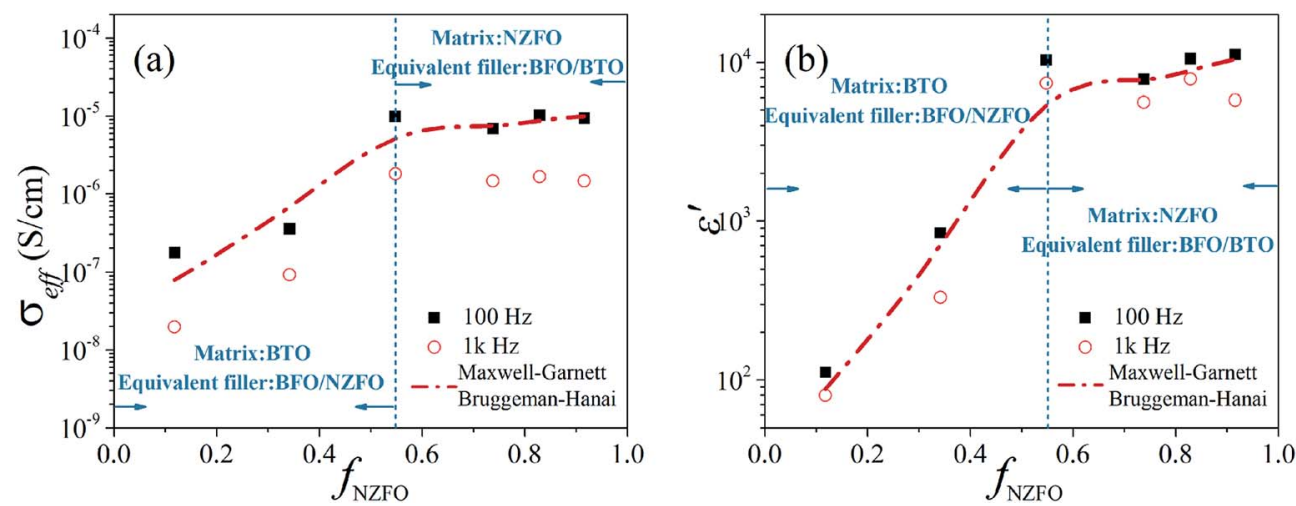

Fig. 5 Variation of (a) the conductivity and (b) effective permittivity of the tri-phase BTO/NZFO/BFO ceramic composite sintered at $1300{ }^{\circ} \mathrm{C}$ for $10 \mathrm{~h}$ as a function of NZFO content $x$ at $100 \mathrm{~Hz}$ and $1 \mathrm{kHz}$ (The hollow-circles and squares represent the experimental data, and the solid/dotdashed line represents the calculated results of Bruggeman equation and Maxwell-Garnett model).

Now the theoretical calculation can be carried out as follows: firstly, the volume fraction of each constituent phase in the composite is obtained by summing up its integral intensity of all peaks in XRD patterns, as shown in Fig. 1a. It indicates that above $f_{\mathrm{c}}$, the volume content of the BFO phase maintains a stable value of about $20-25 \%$.

Secondly, below $f_{\mathrm{c}}$, the BTO exists as the matrix, while the $\mathrm{NZFO/BFO} \mathrm{is} \mathrm{assumed} \mathrm{to} \mathrm{be} \mathrm{the} \mathrm{equivalent} \mathrm{filler.} \mathrm{On} \mathrm{the}$ contrary, above $f_{\mathrm{c}}$, the NZFO is assumed as the matrix, and the $\mathrm{BTO} / \mathrm{BFO}$ exists as the equivalent filler. As mentioned above, we consider the $\mathrm{NZFO} / \mathrm{BFO}$ and $\mathrm{BTO} / \mathrm{BFO}$ as a whole below and above $f_{\text {c. The NZFO/BFO and BTO/BFO equivalent fillers possess }}$ a core-shell structure, where the BFO is seen as the shell. Besides, for the core-shell structure, the Maxwell-Garnett model (M-G model) is used to calculate its effective dielectric constant, which is expressed as follows: ${ }^{13,15}$

$$
\begin{aligned}
& K=K_{1}\left[1+3 f_{2} \beta /\left(1-f_{2} \beta\right)\right] \\
& \beta=\left(K_{2}-K_{1}\right) /\left(K_{2}+2 K_{1}\right)
\end{aligned}
$$

where $K, K_{1}, K_{2}$ are the properties of the composite, the matrix and the filler, respectively. $f_{2}$ is the volume fraction of the filler phase. Thus, for the tri-phased ceramic composite, below $f_{c}$, the effective dielectric constant of the filler NZFO/BFO is available (BTO is the matrix), and above $f_{\mathrm{c}}$, the effective dielectric constant of the filler BTO/BFO can also be obtained (NZFO is the matrix). The parameters used in the calculation of the properties of the composite are summarized in Table 1.
Finally, we use the $\mathrm{B}-\mathrm{H}$ model to calculate the effective dielectric constant of the tri-phased ceramic composite by putting the values of the matrix and the equivalent filler under different $f$ (volume content of NZFO) into the formula as listed in Table 1.

The calculation result is shown in Fig. 5b, in which the red line represents the prediction of $\mathrm{M}-\mathrm{G}$ model and $\mathrm{B}-\mathrm{H}$ model. Obviously, the calculated results that employ the M-G model as well as B-H model fit well with the experimental data at $100 \mathrm{~Hz}$ and $1 \mathrm{kHz}$ as shown in Fig. 5b. The physical model proposed above can also be applied to describe the behavior of the electrical conductivity of the composite as shown in Fig. 5a, which also fits well with our experimental data. It should be noted that the ceramic composite synthesized via a liquid coating process could form a core/shell structure first, nevertheless a higher sintering temperature such as $1300{ }^{\circ} \mathrm{C}$ would destroy the core/ shell structure. It is speculated that there may exist a concentration gradient in the ceramic composite, which means that the semiconductive NZFO was surrounded by Ba-rich layer, resulting in the obstruction of the formation of electric channels and constitution of micro-capacitances (situation similar to the structure of boundary layer capacitor). As a consequence, the series-parallel connection of micro-capacitances is physically responsible for the remarkable increase in the effective permittivity of the composite in terms of equivalent circuit. As shown in Fig. 1a, the content of BTO in the ceramic composite declined and the content of BFO increased with increasing

\begin{tabular}{|c|c|c|c|c|c|c|c|c|}
\hline$f_{\mathrm{NZFO}}$ & Matrix 1 & Filler 1 & $\begin{array}{l}\text { Effective permittivity } \\
\text { (M-G) }\end{array}$ & $\begin{array}{l}\text { Electrical conductivity } \\
\left(\mathrm{S} \mathrm{cm}^{-1}\right)(\mathrm{M}-\mathrm{G})\end{array}$ & Matrix & $\begin{array}{l}\text { Equivalent filler } \\
\text { (matrix 1/filler 1) }\end{array}$ & $\begin{array}{l}\text { Effective permittivity } \\
(\mathrm{B}-\mathrm{H})\end{array}$ & $\begin{array}{l}\text { Electrical conductivity } \\
\left(\mathrm{S} \mathrm{cm}^{-1}\right)(\mathrm{B}-\mathrm{H})\end{array}$ \\
\hline 0.118 & $\mathrm{BFO}$ & NZFO & 22 & $2 \times 10^{-8}$ & BTO & NZFO/BFO & 88 & $9 \times 10^{-8}$ \\
\hline 0.342 & NZFO & BFO & 6006 & $6 \times 10^{-6}$ & BTO & NZFO/BFO & 615 & $6 \times 10^{-7}$ \\
\hline 0.548 & NZFO & $\mathrm{BFO}$ & 8583 & $9 \times 10^{-6}$ & BTO & $\mathrm{NZFO/BFO}$ & 8316 & $8 \times 10^{-6}$ \\
\hline 0.738 & BTO & $\mathrm{BFO}$ & 45 & $5 \times 10^{-8}$ & NZFO & BTO/BFO & 7316 & $7 \times 10^{-6}$ \\
\hline 0.829 & $\mathrm{BFO}$ & BTO & 20 & $2 \times 10^{-8}$ & NZFO & $\mathrm{NZFO/BFO}$ & 8931 & $9 \times 10^{-6}$ \\
\hline 0.916 & $\mathrm{BFO}$ & ВТО & 18 & $2 \times 10^{-8}$ & NZFO & BTO/BFO & 10492 & $1 \times 10^{-5}$ \\
\hline
\end{tabular}

Table 1 Parameters used in the calculation of the properties of the composite 
NZFO content. BFO, a commonly used microwave absorbing material, possesses high resistivity and relatively small effective permittivity compared with BTO. Hence, with the enlargement of $x$, although the thinning of dielectric layer between NZFO makes the term of $\left|\frac{f_{\mathrm{c}}-f}{f_{\mathrm{c}}}\right|^{-q}$ (eqn (2)) larger, the increment of BFO in the dielectric layers results in the diminution of the whole effective permittivity in the dielectric layer $\left(\varepsilon_{0}\right.$ in eqn (2)), which synergistically make the permittivity to exhibit an almost composition-independent behavior in a wide composition range above the percolation threshold compared with the case in traditional percolative composites. Therefore, the engendering of a third phase (BFO) compensates the nonlinear increase in the dielectric properties of such percolative composite.

Fig. 6 shows the magnetic hysteresis loops of the tri-phase $\mathrm{BTO} / \mathrm{NZFO} / \mathrm{BFO}$ composite with various $x$ measured at room temperature, in which the enlarged low field region for $x=0.9$ is shown as inset. The saturation magnetization of the composite decreases with decreasing ferrite content from $73.2 \mathrm{emu} \mathrm{g}^{-1}$ at $x$ $=0.9$ to 36.8 emu g $^{-1}$ at $x=0.7$. For $x=0.9$, the coercivity of the composite is about 50 Oe, larger than that of conventional BTO/ NZFO composite, ${ }^{29}$ indicating that the formation of the BFO phase with a high coercivity of $>50$ Oe (ref. 30 ) may cause an increment in the coercivity of the tri-phase system to some extent.

The saturation magnetization is mainly originated from the ferrite phase, and it is closely dependent on the parameters such as grain size and density following the expression of: ${ }^{31}$

$$
M_{\mathrm{s}}=(1-p) \delta_{\mathrm{s}} d_{\mathrm{s}}
$$

where $p$ is the porosity of the composite, $d_{\mathrm{s}}$ the grain size of NZFO particles, and $\delta_{\mathrm{s}}=M_{\mathrm{s}}$ (obs) $/ M_{\mathrm{s}}$ (sat). It can be seen that the saturation magnetization will increase if the grain size and the density of the composite get enhanced. This can explain the variation of $M_{\mathrm{s}}$ values versus increasing NZFO content as shown in Fig. 6, because the grain size and density are the largest for $x=0.9$.

The frequency dependence of the effective permeability of the tri-phase BTO/NZFO/BFO composite on ferrite content $x$ is

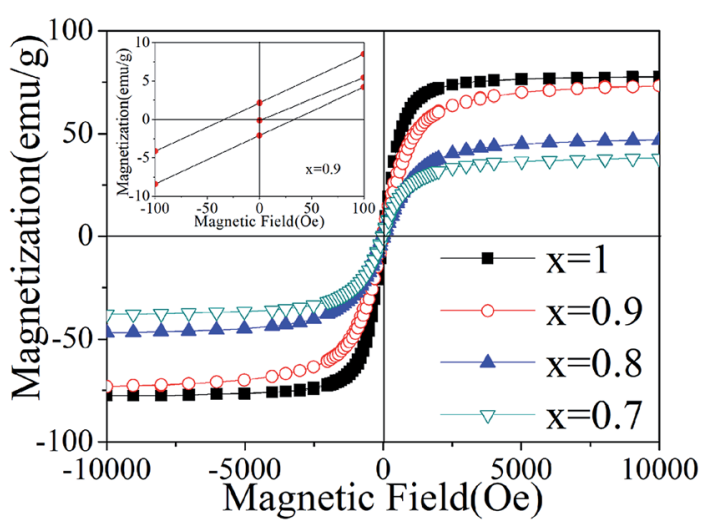

Fig. 6 Room temperature magnetic hysteresis loops of the tri-phase $\mathrm{BTO} / \mathrm{NZFO} / \mathrm{BFO}$ ceramic composites sintered at $1300^{\circ} \mathrm{C}$ for $10 \mathrm{~h}$. The inset shows the low field region of $x=0.9$. shown in Fig. 7a. As is expected, the effective permeability increases with increasing ferrite content in accordance with the composite law. The cut-off frequencies are found to be above 20 $\mathrm{MHz}$, which broadens the applicability of the composite in a wide frequency region. It is also noticed that the value of effective permeability is much smaller than that of the BTO/ NZFO system. ${ }^{29}$

In fact, two factors should be considered in understanding the decrease of saturation magnetization and effective permeability with decreasing NZFO content. One is the composite effect, that is, the introduction of nonmagnetic BTO and hardmagnetic BFO phase weakens the interactions and continuum among the NZFO crystalline particles. As a result, the initial permeability decreases due to lower ferrite content and further weakening effect of other two constituent phases (BTO and $\mathrm{BFO})$. It can be deduced that the magnetic response of such triphase composite can be modulated by controlling ferrite content in the system. The other is the emergence of demagnetizing filed that usually exists between the interfaces of the constituent phases according to grain boundary demagnetizing field theory, which can be written as: ${ }^{32}$

$$
\mu_{\mathrm{app}}=\frac{\mu_{\mathrm{i}}}{1+0.75 \frac{t}{D} \frac{\mu_{\mathrm{i}}}{\mu_{\mathrm{L}}}}
$$

where $\mu_{\text {app }}$ is the apparent initial permeability of the composite considering the effect of demagnetizing field, $\mu_{\mathrm{b}}$ the permeability of grain boundaries, the effective thickness of grain boundaries, $D$ the average grain size. In as-prepared tri-phase composite, the amount of interfaces is comparably larger in structure, thus the demagnetizing effect will impose a more observable decrease on the apparent permeability of the composite. The demagnetizing field is mainly induced by nonmagnetic BTO phase and the formation of BFO would limit the growth of NZFO because a portion of ferrites are consumed as Fe-source to form BFO in the system.

The plots of the initial permeability of the composite as a function of NZFO content $\left(f_{\text {NZFO }}\right)$ are shown in Fig. 7b. For simplifying analysis, the BTO and BFO phase are considered wholly as dielectric matrix while only NZFO phase is thought as magnetic filler. The theoretical calculation can be conducted using the aforementioned Maxwell-Garnett model (eqn (4)) and Bruggeman-Hanai approximation (eqn (3)). The calculation results are illustrated in Fig. $7 \mathrm{~b}$, in which the solid line represents the results of Bruggeman equation while the dot-dashed line represents the prediction of $\mathrm{M}-\mathrm{G}$ model.

The calculated values from M-G equation agree well with the experimental values when the NZFO content is below $30 \%$. However, obvious deviation occurs between the measured values and theoretical predictions at higher NZFO content above $50 \%$. It is known that the M-G theory can only be applied in the situation where all the interactions between the filler particles are ignored, while the Bruggeman equation takes the local influence of adjoining particles into consideration and treats the constituent phases in the composite equally. Hereby we assume that the BFO phase is as homogenous as the BTO phase in controlling the magnetic properties of the composite, 

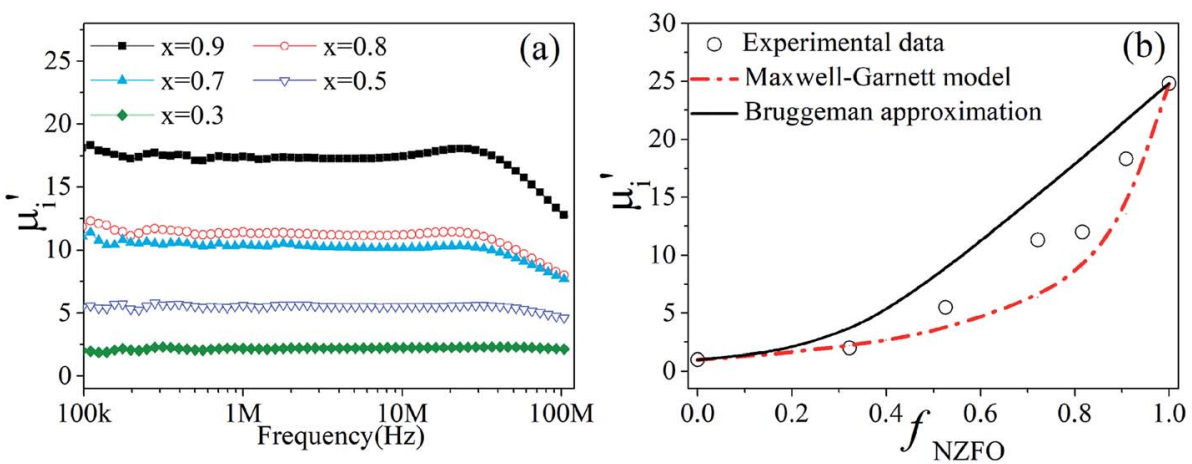

Fig. 7 (a) Frequency dependence of the effective permeability and (b) composition dependence of the initial permeability of the tri-phase BTO/ NZFO/BFO composite at the frequency of $10 \mathrm{MHz}$. The open circles represent the experimental data, and the solid/dot-dashed line represents the calculated results of Bruggeman equation and Maxwell-Garnett model, respectively.

thus the small difference between BFO and BTO can be neglected. When the NZFO content is below $50 \%$, the ferrite particles form dispersive microstructure in the BTO matrix. This situation fits well with the description of M-G model. Therefore, a good agreement between the experimental results and theoretical predictions is observed. However, as the NZFO content increases, the filler particles begin to contact with one another and finally form percolating topological microstructure, causing the interactions to occur between the interfaces of constituent phases inevitably. Obviously, the effect of such interactions will become dominant in the composite above $x=$ 0.5. Although the ceramic composites were prepared by liquid coating method and a core/shell structure was formed during the preparation process, the elemental interdiffusion will get serious and the third constituent phase will damage the core/ shell structure at a high sintering temperature of $1300{ }^{\circ} \mathrm{C}$, leading to a structure which is more close to the situation in which the Bruggeman equation will be more suitable. Consequently, the experimental points fall between the theoretical values of the M-G model and the Bruggeman equation. It is worth noting that BFO may help to isolate the magnetic NZFO particles, preventing them to get aggregating with neighbouring particles. Due to this effect, the majority of the experimental points tend to fall onto the M-G curve instead of the Bruggeman curve as shown in Fig. 7 b.

On the whole, in our tri-phase composite, the effective permittivity can keep a stable high value of $\sim 10000$ (Zhang's group reported a $\mathrm{BaTiO}_{3}-\mathrm{Ni}_{0.2} \mathrm{Cu}_{0.2} \mathrm{Zn}_{0.6} \mathrm{Fe}_{2} \mathrm{O}_{4}$ system with a permittivity lower than 40 ; the $\mathrm{Bi}_{4} \mathrm{Ti}_{3} \mathrm{O}_{12} / \mathrm{Ni}_{0.25} \mathrm{Cu}_{0.2} \mathrm{Zn}_{0.55^{-}}$ $\mathrm{Fe}_{2} \mathrm{O}_{4}$ system posseses permittivity of as low as 170$)^{7,33}$ above $50 \%$ ferrite loading. The plateau of both conductivity and permittivity between $50 \%$ and $90 \%$ filler content contributes to the stability of performance within a wide composition range. It suggests that after being increased to an expected high value of permittivity with the help of percolation effect, introducing lowdielectric phase into the composite could help compensate the nonlinear increase in dielectric properties. The findings of this work offer us a promising way to prevent electrical properties from drastic change with varying composition around the percolation threshold. The tri-phase ferroelectric/ferromagnetic composite is thus of great applicable interest in advanced electronic devices due to its stable giant permittivity and extremely high permeability.

\section{Conclusions}

The as-prepared BTO/NZFO ceramic composites exhibits a larruping dielectric response combined by the percolation effect with a percolative threshold larger than 0.5 and the existence of the BFO phase, which has a lower permittivity compared with the BTO matrix. When the volume fraction of NZFO is less than $50 \%$, the behavior of the composite is similar to that of conventional percolative composites, however, when the ferrite content becomes larger than $50 \%$, the permittivity preserves a high value of approximately 10000 owing to the existence and adjustment effect of the BFO phase. The formation of the BFO phase did not jeopardize the dielectric properties of the ceramic composites, on the contrary, the permittivity can be nailed at a stable value through appropriate adjustment of the volume fraction of the BFO phase. The phase transformation makes the effective permittivity of the insulating layers much lower and hence helps the composite exhibit stable properties. The BTO/ $\mathrm{BFO} / \mathrm{NZFO}$ ternary ceramic composite is characterized by its impressive dielectric properties and may become a promising candidate for next-generation electronic device applications.

\section{Conflicts of interest}

There are no conflicts to declare.

\section{Acknowledgements}

This work was supported by the Natural Science Foundation of China under grant no. 51772269 and 51802345.

\section{References}

1 C. Israel, N. D. Mathur and J. F. Scott, Nat. Mater., 2008, 7, 93-94.

2 W. Eerenstein, N. D. Mathur and J. F. Scott, Nature, 2006, 442, 759-765. 
3 C. A. Vaz, J. Hoffman, C. H. Ahn and R. Ramesh, Adv. Mater., 2010, 22, 2900-2918.

4 N. M. Triet, T. Q. Trung, N. T. D. Hien, S. Siddiqui, D.-I. Kim, J. C. Lee and N.-E. Lee, Nano Res., 2015, 8, 3421-3429.

5 Y. Tang, Y. Zhang, G. Ma, N. Ma and P. Du, J. Phys. Chem. C, 2015, 119, 17995-18005.

6 Y. Tang, Y. Zhang, N. Ma and P. Du, J. Mater. Chem. C, 2015, 3, 9076-9088.

7 H. Zhong and H. W. Zhang, J. Magn. Magn. Mater., 2005, 288, 74-78.

8 A. L. Efros, Phys. Rev. B: Condens. Matter Mater. Phys., 2011, 84.

9 C. Pecharromán, F. Esteban-Betegón, J. F. Bartolomé, S. López-Esteban and J. S. Moya, Adv. Mater., 2001, 13, 1541-1544.

10 C. Pecharromán, F. Esteban-Betegón and R. Jiménez, Ferroelectrics, 2010, 400, 81-88.

11 C. J. Won, R. C. Kambale and N. Hur, J. Alloys Compd., 2011, 509, 6969-6972.

12 C.-W. Nan, Y. Shen and J. Ma, Annu. Rev. Mater. Res., 2010, 40, 131-151.

13 H. Zheng, Y. L. Dong, X. Wang, W. J. Weng, G. R. Han, N. Ma and P. Y. Du, Angew. Chem., Int. Ed. Engl., 2009, 48, 89278930.

14 B. Xiao, Y. L. Dong, N. Ma and P. Y. Du, J. Am. Ceram. Soc., 2013, 96, 1240-1247.

15 J. Q. Huang, P. Y. Du, L. X. Hong, Y. L. Dong and M. C. Hong, Adv. Mater., 2007, 19, 437-440.

16 B. Xiao, W. Zheng, Y. L. Dong, N. Ma and P. Y. Du, J. Phys. Chem. C, 2014, 118, 5802-5809.

17 Q. Chen, P. Y. Du, L. Ji, W. J. Weng and G. R. Han, Appl. Phys. Lett., 2007, 91, 22912.
18 Z. H. Chen, J. Q. Huang, Q. Chen, C. L. Song, G. R. Han, W. J. Weng and P. Y. Du, Scr. Mater., 2007, 57, 921-924.

19 R. C. Pullar, Prog. Mater. Sci., 2012, 57, 1191-1334.

20 R. M. Almeida, W. Paraguassu, D. S. Pires, R. R. Corrêa and

C. W. de Araujo Paschoal, Ceram. Int., 2009, 35, 2443-2447.

21 D. Suastiyanti, A. Sudarmaji and B. Soegijono, AIP Conf. Proc., 2012, 1454, 238-241.

22 C. C. Yang, Y. J. Gung, C. C. Shih, W. C. Hung and K. H. Wu, J. Magn. Magn. Mater., 2011, 323, 933-938.

23 Y. Wang, Y. Huang and Q. F. Wang, J. Magn. Magn. Mater., 2012, 324, 3024-3028.

24 M. T. Buscaglia, V. Buscaglia, L. Curecheriu, P. Postolache, L. Mitoseriu, A. C. Ianculescu, B. S. Vasile, Z. Zhe and P. Nanni, Chem. Mater., 2010, 22, 4740-4748.

25 B. Xiao, J. C. Wang, N. Ma and P. Y. Du, Advances in Multifunctional Materials and Systems II: Ceramic Transactions, 2014, vol. 245, pp. 23-34.

26 B. Xiao, W. Zheng, M. K. Zhu, W. J. Zhao, N. Ma and P. Y. Du, J. Mater. Chem. C, 2014, 2, 7482.

27 Z. J. Wang, W. Y. Zhou, X. Z. Sui, L. N. Dong, H. W. Cai, J. Zuo, X. R. Liu and Q. G. Chen, Polym. Compos., 2018, 39, 887-894.

28 H. Zheng, L. Li, Z. J. Xu, W. J. Weng, G. R. Han, N. Ma and P. Y. Du, J. Phys. D: Appl. Phys., 2013, 46, 185002.

29 B. Xiao, Y. Tang, G. D. Ma, N. Ma and P. Y. Du, Appl. Phys. A, 2015, 119, 1291-1300.

30 R. E. El Shater, E. H. El-Ghazzawy and M. K. El-Nimr, J. Alloys Compd., 2018, 739, 327-334.

31 R. V. Mangalaraja, S. Ananthakumar, P. Manohar and F. D. Gnanam, Mater. Lett., 2003, 57, 2666-2669.

32 H. Rikukawa, IEEE Trans. Magn., 1982, 18, 1535-1537.

33 W. W. Ling, H. Nie, Y. X. Li and H. W. Zhang, J. Alloys Compd., 2012, 525, 120-125. 